\title{
10. COMMISSION DES PHENOMENES PHOTOSPHERIQUES
}

\author{
Président: L. d'Azambuja.
}

Président d'Honneur: W. Brunner†.

Membres: MM. Abetti, Alfvén, Cardús, Chapman, Cimino, Contino, Coutrez, Das, Djurkovic, Eigensson, Ferraro, Gullón, Kiepenheuer, Kopecký, Krat, Macris, Mergentaler, H. Müller, Mustel, Newton, Nicholson, Notuki, Ohman, Roberts, Romañá, Rubashev, A. H. Shapley, Mme Slonim, MM. Smerd, Stepanov, TandbergHanssen, Waldmeier, Wayman.

\section{OBSERVATIONS ET PUBLICATIONS COURANTES}

La période écoulée depuis la dernière Assemblée générale de l'Union astronomique internationale est caractérisée par une augmentation très sensible des stations équipées pour la photographie de la photosphère solaire. Cette augmentation est due, en premier lieu, aux recommandations du Comité spécial de l'Année géophysique internationale qui, dès ses premières réunions, a prescrit d'intensifier les observations du Soleil. En second lieu. elle atteste les premiers effets des circulaires envoyées par l'Observatoire fédéral de Zurich dans le but de réaliser, comme il l'avait proposé dès I948, l'étude détaillée en commun de l'évolution des taches solaires. A l'heure actuelle, trente stations environ, instituts astronomiques ou astronomes amateurs, sont engagés dans cette étude, dont les résultats seront centralisés à Zurich. Afin d'assurer l'homogénéité nécessaire aux observations et reconnaître éventuellement leurs lacunes, une première période comprise entre le I6 juin I957 (o heure T.U.) et le 30 juin ( 24 heures) a été fixée au cours de laquelle les observateurs ont envoyé leurs photographies à Zurich. L'étude de ces documents, que suivront des recommandations appropriées, est actuellement en cours.

Les prescriptions du Comité de l'Année géophysique ont eu une autre conséquence: c'est d'engager les observatoires à dresser des cartes journalières du Soleil, sur lesquelles sont représentés, non seulement les taches, mais aussi les principaux phénomènes chromosphériques et coronaux; les contours des facules y sont tracés d'après les spectrohéliogrammes, qui ont le grand avantage de les montrer sur tout le disque, depuis leur lever jusqu'à leur coucher.

A l'heure actuelle, de telles cartes sont établies à l'Institut Fraunhofer, à Moscou, à Meudon et à Mitaka (Tokio). Pour éviter les lacunes dans les observations, les deux premiers établissements se sont assurés le concours d'autres stations situées dans des régions du Globe différentes; mais l'envoi des cartes est ainsi retardé. A Meudon, il a paru préférable d'utiliser les seuls documents obtenus à l'Observatoire, et à celui du Pic du Midi pour la couronne, et d'expédier les cartes le soir même de l'observation. On peut remarquer d'ailleurs que, si une douzaine d'observatoires, convenablement répartis en longitude, étaient chargés d'établir et de distribuer des cartes par ce second procédé à ceux qui en feraient la demande, les lacunes des observations seraient, pour ces derniers, à peu près automatiquement comblées. En outre, la demande de certains géophysiciens, notamment de $S$. Chapman, que les caractères de l'activité solaire leur soient communiqués plusieurs fois par jour, serait ainsi satisfaite, de par la dispersion même, en longitude, des observatoires participants. Toutefois, pour que la méthode ait toute son efficacité, il serait nécessaire que les cartes soient construites sur le même plan, comportent les mêmes conventions, soient tracées à la même échelle, afin d'assurer la comparaison dans les meilleures conditions possibles. En fait si, à l'origine, les cartes établies sur l'initiative des établissements précités présentaient de grosses divergences, celles-ci se sont atténuées peu à peu et, à l'heure actuelle, les représentations sont peu différentes les unes des autres.

En dehors des cartes journalières, les cartes héliographiques par rotation ont continué à être dressées et publiées par les observatoires qui en avaient antérieurement assumé la 


\section{COMMISSION 10}

charge: Zurich (Heliographische Karten der Photosphäre); Uccle (Cartes publiées dans Ciel et Terre et communiquées aux principaux observatoires solaires); Mitaka (Synoptic maps of sunspots, $H \alpha$ flocculi and dark filaments). A ces établissements, il faut ajouter l'Observatoire de Kanzelhöhe qui, en coopération avec l'Institut d'Astrophysique de Paris, a commencé la publication de cartes planisphères des taches et de phénomènes chromosphériques dans Documentation des observateurs.

Le Royal Greenwich Observatory a complété la publication des Photoheliographic Results jusqu'à la fin de r949. Les fascicules relatifs aux années r95o à r955 sont en cours d'impression. Par ailleurs, les positions héliographiques des taches, leur aire, les tables d'évolution des groupes, sont l'objet de déterminations de plus en plus nombreuses publiées dans divers recueils. Citons notamment: les U.S. Naval Observatory Circulars, les Publikationen der Eidg. Sternwarte de Zurich, les notes des observatoires d'Arcetri et de Monte-Mario dans les Memorie della Società astronomica italiana (depuis le Ier janvier I958, les observations du Monte-Mario font l'objet d'une publication indépendante), celles de l'Observatoire d'Uccle dans Ciel et Terre; les Monthly Bulletins of solar phenomena, de Mitaka et de la section astronomique de l'Université américaine de Beyrouth; le Bulletin héliophysique de l'Observatoire de l'Ebre.

Le nombre d'établissements déterminant les nombres relatifs de Wolf a augmenté, lui aussi, dans des proportions notables et la tâche de l'Observatoire de Zurich, chargé de les centraliser pour leur publication dans différentes revues et dans le Quarterly Bulletin on Solar Activity, s'en est trouvée accrue. L'Observatoire fédéral détermine toujours, en outre, des nombres relatifs provisoires avec la coopération des stations d'Arosa et de Locarno, pour une publication rapide. Des déterminations analogues, résultant des observations de plusieurs instituts américains, sont publiées dans les Bulletins $d u$ C.R.P.L. D'autre part, depuis quelques années, des nombres de Wolf quotidiens sont incorporés dans les messages ursigrammes relatifs à l'activité chromosphérique. Ils sont établis par les stations qui préparent les ursigrammes. Cependant, avec la forte reprise de l'activité solaire depuis I955, les divergences entre les valeurs de ces nombres, pour le même jour d'observation, déjà signalées auparavant, se sont manifestées de nouveau. Il semble donc, dans ces conditions, qu'il serait désirable d'étudier, comme on l'avait envisagé à Dublin, lors de la dernière Assemblée générale de l'Union, la possibilité d'adjoindre à ces nombres un autre indice d'activité basé, par exemple, sur une estimation rapide de l'aire des taches, pour comparer la précision des deux méthodes et, finalement, conserver la meilleure.

\section{TRAVAUX DE RECHERCHES}

\section{Taches}

\section{Distribution. Statistique}

H. W. Newton et A. S. Milsom[r] ont étudié systématiquement le rapport entre les surfaces tachées dans les hémisphères nord et sud du Soleil et les variations de ce rapport dans le temps. Ils ont utilisé pour cela la documentation photographique de Greenwich sur sept cycles undécennaux, en la complétant par des données antérieures sur quatre cycles. Ils concluent que la proportion des taches dans les deux hemisphères subit une variation qui ne paraît pas relever du hasard, mais qui n'est pas non plus périodique. En aucun cas, cette variation n'apparaît liée au cycle de 22 ans, auquel se rapporte la polarité des taches. G. G. Mursalinova [2] a fait un travail analogue en complétant la documentation de Greenwich par les relevés de Tachkent.

Dans une série d'articles, M. Kopecký [3] propose quelques hypothèses simplificatrices qui lui permettent d'établir des formules à partir desquelles il est possible de déterminer les nombres de groupes de taches formés respectivement dans l'hémisphère visible et dans l'hémisphère invisible, ainsi que leur durée de vie. Il présente des courbes et un nomogramme qui facilitent la détermination. Ses résultats paraissent en assez bon accord avec l'observation.

Le même auteur revient d'autre part $[4,5]$ sur la fonction de visibilité des taches selon 


\section{PHENOMENES PHOTOSPHERIQUES}

les distances croissantes au centre du disque solaire, et les diverses formules proposées pour la représenter. Selon lui, aucune de ces formules n'est pleinement satisfaisante. M. Hotinli [6] conteste ce point de vue et estime que la relation indiquée par lui en I95I :

$$
\cos l(\mathrm{x}-0.075 \operatorname{tg} l) \text {, }
$$

traduit bien l'effet de raccourcissement dû à la perspective. Remarquons en passant que le désaccord porte sur d'assez petites quantités et qu'il ne faut pas perdre de vue que, au voisinage du bord, la principale cause d'erreur dans les mesures réside surtout dans la turbulence atmosphérique terrestre, qui trouble et agite les images.

Avant de clore ce paragraphe, signalons qu'au cours d'une étude de la formation des groupes de taches portant sur plus de trois cycles undécennaux, Mme M. d'Azambuja [7], s'aidant des facules chromosphériques pour identifier plus sûrement les groupes nouveaux se formant au voisinage de groupes plus anciens, a trouvé qu'il se forme environ deux fois plus de groupes à l'est du Soleil qu'à l'ouest. En outre ces groupes seraient, en moyenne, plus durables. C'est là un fait d'observation à peu près incontestable; il reste évidemment à lui trouver une explication satisfaisante. On sait que des dissymétries analogues, mais moins importantes, ont été à plusieurs reprises signalées antérieurement.

\section{Structure et évolution des taches}

E. Jensen, J. Nordö, et T. S. Ringnes [8], ont recherché si l'on peut déceler des variations systématiques dans le temps du rapport $q$ entre l'aire totale des taches, ombre et pénombre, et leur ombre seule. Ils ont utilisé pour cela la documentation de Greenwich entre 1878 et I945, en ne retenant que les mesures faites à moins de 0.650 rayon solaire du centre du disque, sur des taches simples régulières, dont le diamètre était au moins égal à $1 /$ roo du rayon solaire. Ils ont sélectionné ainsi 653 taches. Malgré de larges variations du rapport $q$, il y a une indication nette que ces variations, dans l'ensemble, suivent le cycle de onze ans. Il semble, d'autre part, que $q$ décroisse en période de maximum avec le rayon des taches. Ces relations subsistent quand, au lieu de taches sélectionnées, on considère toutes les taches ou des taches récurrentes.

E. Tandberg-Hanssen [9] a étendu cette étude aux groupes bi-polaires ou composites. Il confirme, dans l'ensemble, les résultats précédents. En outre, il trouve que $q$ paraît indépendant de l'écartement des composantes du groupe, quand celui-ci dépasse $3^{\circ}$.

W. Gleissberg [ro], examinant la classification de Zurich au point de vue du stade de développement des taches estime que, si la classe $\mathrm{J}$ était associée à la classe $\mathrm{C}$, ce système de classification serait pratiquement parfait.

\section{Rotation du Soleil, d'après les positions des taches et des facules}

M. Waldmeier [n] a mesuré les positions successives sur le disque d'une tache qui, malgré la phase déjà avancée du cycle solaire actuel, s'est montrée à la latitude $+48^{\circ}$ du I $_{5}$ au $3 \mathrm{I}$ août 1956 . Il a trouvé pour sa vitesse angulaire de rotation $\xi=12^{\circ} 35$. La loi de rotation de Greenwich aurait donné pour la même latitude $\xi=13^{\circ} \circ 7$. Peut-être s'agit-il d'une tache exceptionnelle. Un groupe à une latitude sensiblement égale, mesurée cinq mois plus tard pendant six jours par M. Kopecký, J. Kvicala et J. Ptáček [r2] a donné $\xi=13^{\circ} 2$.

M. Waldmeier [13], ayant remarqué que, de I95I à I954, de nombreuses petites facules étaient visibles près des pôles pendant deux ou trois jours à une latitude moyenne de $66^{\circ}$, les a utilisées pour obtenir des mesures de la vitesse de rotation solaire dans ces régions dépourvues de taches; l'auteur a trouvé, entre les parallèles $70^{\circ}$ et $80^{\circ}$, des valeurs de $\xi$ comprises entre $9: 7$ et $10^{\circ} 5$.

De son côté, Mlle K. Milosević [14] a identifié ces facules, pour les mêmes jours d'observation, avec des facules chromosphériques visibles sur les spectrohéliogrammes $\mathrm{K}_{3}$ de Meudon. Leur diamètre était seulement un peu plus grand. Elle a reconnu ensuite que des facules semblables existaient sur tout le disque, jusqu'à l'équateur. Ayant mesuré leurs positions successives, elle en a déduit:

$$
\xi=14^{\circ}: 10-3^{\circ} \cdot 23 \phi^{2} \text {, }
$$




\section{COMMISSION 10}

la formule parabolique étant plus appropriée, aux hautes latitudes, que la formule en $\sin ^{2} \phi$ de Carrington. A la latitude $75^{\circ}$, la valeur de $\xi$ serait ainsi de $8^{\circ} 7$.

U. Becker [15], reprenant une idée antérieurement émise a étudié, à l'aide des Photoheliographic Results de 1879 à I94I, les concentrations de taches dans des régions de longitudes privilégiées, qui ont une existence en général beaucoup plus longue que la durée de vie d'un groupe isolé. De telles concentrations peuvent marquer l'existence de phénomènes sous-jacents générateurs de taches et dont il est intéressant de connaître la vitesse de rotation. L'auteur a reconnu 46 foyers distincts dont la durée était comprise entre 6 et 2 I rotations. Leur répartition en longitude et dans le temps a paru n'obéir à aucune loi. Leur vitesse de rotation a dépassé de $2^{\circ}$ à $3^{\circ}$, pour une révolution complète du Soleil, la vitesse des taches individuelles à la même latitude. Par contre, le ralentissement polaire ne paraît pas très différent.

\section{Périodicité}

Le cadre restreint de ce rapport ne permet pas d'analyser les nombreuses recherches effectuées dans le but de mettre en évidence, à l'aide des surfaces tachées ou des nombres de Wolf les particularités qui caractérisent ou distinguent les uns des autres les cycles successifs de l'activité solaire. Bornons-nous à citer les travaux de W. Gleissberg [16] sur l'asymétrie de la courbe d'activité dans le cycle; les études du même auteur [r7], ainsi que de S. B. Nicholson [18], J. M. Slonim et K. F. Kuleshova [19], T. Fortini [20], P. M. Djurković [21], V. F. Christjakov[22], L. Arbey [23], U. I. Vitinsky et B. M. Rubashev [24], J. Xanthakis [25], pour mettre en évidence et discuter les fluctuations que les courbes subissent dans leurs différentes parties au cours des cycles successifs. Notons encore la recherche de périodes plus longues que le cycle de onze ans, qui paraît décidément faire ressortir l'existence d'un cycle de 78 à 80 ans (M. Waldmeier [26], D. J. Schove [27], W. Gleissberg [28], M. Dizer [29]); la description de méthodes conduisant à établir des formules à un ou plusieurs paramètres qui permettent, dans une certaine mesure, de prévoir l'allure et la durée du cycle undécennal (B. Thuring [3०], M. Ogawara et K. Tomatsu [31], M. Kopecký [32], W. Gleissberg [33]); enfin, un travail de W. Gleissberg [34] qui, faisant intervenir les saisons terrestres, semble indiquer une certaine prépondérance des grands groupes de taches en été.

\section{Travaux théoriques}

H. Alfvén [35] montre que la formation des taches ne peut valablement être imputée à la rotation différentielle du Soleil, comme le supposent plusieurs auteurs. Par ailleurs, il réfute certaines assertions de T. G. Cowling, qui a discuté sa théorie magnéto-hydrodynamique des taches.

Se référant à la théorie d'Alfvén, K. P. Chopra [36] étudie comment ses ondes magnétohydrodynamiques peuvent se transmettre d'un milieu à un autre, séparé du premier par un espace vide de très faible épaisseur.

Selon D. H. Menzel, D. Layzer et M. Krook [37], une étoile à rotation différentielle et à champ magnétique permanent comme le Soleil est le siège d'oscillations de torsion qui changent de sens cycliquement. Les auteurs, utilisant les données connues sur le Soleil, concluent que leur hypothèse est en bon accord avec le cycle de 22 ans.

E. N. Parker [38], admettant l'existence de tubes horizontaux de flux magnétique dans l'atmosphère solaire conductrice et d'un champ toroîdal immédiatement sous la photosphère, en déduit la formation des taches avec leurs caractères principaux, notamment leur orientation est-ouest, leur bi-polarité et le renversement de celle-ci à chaque cycle.

\section{Divers}

V. E. Stepanov et M. A. Kljakotko [39] ont étudié les mouvements à grande échelle dans les couches sub-photosphériques à l'aide des déplacements des taches. W. A. Miller [40], à l'aide d'enregistrements cinématographiques, a recherché comment variait l'intensité dans les taches et fait quelques remarques sur un travail analogue publié 


\section{PHENOMENES PHOTOSPHERIQUES}

antérieurement par R. Ananthakrishnan (Trans. I.A.U. 9, I27). V. E. Stepanov[41] a déterminé la température effective de l'ombre et de la pénombre des taches et a trouvé respectivement, avec une bonne approximation, $3920^{\circ}$ et $5290^{\circ}$.

\section{Granulation}

G. Thiessen [42] a effectué de nouvelles observations visuelles avec l'objectif de $60 \mathrm{~cm}$ de Hambourg, souvent diaphragmé, mais parfois à toute ouverture. Il confirme la valeur de $I^{\prime \prime}$ à $2^{\prime \prime}$ pour le diamètre des grains. Ceux-ci seraient plutôt polygonaux que circulaires. A $\lambda 5_{550} \AA$, leur éclat surpasserait celui du fond de $35 \%$. V. A. Krat et N. M. GoldbergRogozinskaya [43] confirment aussi leurs résultats antérieurs selon lesquels le vrai diamètre des grains, compte tenu de la diffraction, serait nettement plus petit.

C. Macris et D. Elias [44] ont recherché s'il n'existait pas de variation dans le temps du diamètre et de l'éclat des granules. La recherche a porté sur une période s'étendant de I880 à I943, mais à l'aide de clichés très espacés obtenus par Janssen, Keenan et Lyot. Il y a peut-être une relation entre le diamètre des granules et le nombre de Wolf. Les auteurs signalent en outre que la granulation de maximum est plus contrastée que celle de minimum. W. A. Miller [45], a recherché lui aussi des variations de même nature, avec un même instrument, mais sur une période de 38 mois seulement. Il n'a trouvé aucun changement appréciable.

A un point de vue plus théorique, F. E. Stuart et J. H. Rush [46] d'une part, A. Skumanich [47], d'autre part, ont appliqué à l'étude de la turbulence et du contraste des grains sur des clichés du Mount Wilson, les méthodes statistiques d'autocorrélation. Quelques désaccords apparaissent entre leurs résultats et ceux que donnent les méthodes d'observation visuelle directes. G. Wlérick [48] estime que ces désaccords sont dus surtout à la part d'interprétation qui intervient toujours avec ces derniers procédés.

Les mesures du diamètre et de l'éclat relatif des granules sont affectées par la limite du pouvoir de résolution des instruments et par la turbulence de notre atmospłıère. $M$. S. Uberoi [49] avait traité ce problème par l'analyse. J. Rösch l'a abordé du point de vue expérimental [so], en associant à la lunette de $23 \mathrm{~cm}$ d'ouverture de l'Observatoire du Pic du Midi où, comme l'on sait, la qualité des images est exceptionnellement bonne, une chambre cinématographique permettant de faire 20 poses par seconde, chacune d'elles pouvant être inférieure à $\mathrm{I} / 300$ de seconde. Les images obtenues révèlent avec quelle étonnante rapidité les détails changent d'aspect, cette remarque étant valable, non seulement pour la granulation, mais aussi à l'échelle des taches et des principaux caractères de leur structure. Quand on sélectionne celles d'entre les images qui sont à peu près identiques entre elles et doivent, par suite, rendre le mieux compte de l'aspect réel des phénomènes, on constate que les granules, dont le diamètre, au centre du disque, est compris entre I" et $2^{\prime \prime}$, sont allongés près du bord, parallèlement à celui-ci. On constate en outre la présence de points brillants dans l'ombre des taches, comme l'avait signalé autrefois le $\mathrm{P}$. Chevalier. La distribution de ces points, qui paraissent plus petits que la limite de résolution, est beaucoup moins régulière que celle des granules photosphériques. Il semble dès lors que la question du rôle de la diffraction soit ainsi résolu. D'une part, en effet, la présence sur la même image des deux sortes de granules montre la différence d'aspect du champ selon que les objects qui le remplissent sont de dimensions supérieures ou inférieures à la limite de résolution. D'autre part, l'allongement des granules parallèlement au bord solaire ne peut être dû qu'à un effet de perspective sur des objets ayant réellement un diamètre de I"5. S'il s'agissait seulement d'une image de diffraction, elle apparaîtrait circulaire aussi bien au bord qu'au centre du disque.

Les effets de la turbulence seraient évidemment très atténués si les photographies pouvaient être prises au-dessus des régions denses de l'atmosphère où elle est produite. Une première tentative a été faite dans ce sens par D. E. Blackwell, D. W. Dewhirst et A. Dollfus [51], qui ont équipé la nacelle d'un ballon libre avec une lunette de $30 \mathrm{~cm}$ d'ouverture et un dispositif photographique permettant des poses de $\mathrm{r} / 5000$ de seconde. La lunette était larguée par parachute avant l'atterissage du ballon. Au cours d'un vol 


\section{COMMISSION 10}

à l'altitude de $6000 \mathrm{~m}$, effectué à partir de l'Observatoire de Meudon, une série d'images dont la qualité est probablement un peu supérieure aux meilleures du Pic du Midi, a été enregistrée. Dans l'ensemble, elles confirment les résultats obtenus dans ce dernier Etablissement. Les granules ont un diamètre moyen voisin de I"2 ; ils ne sont pas circulaires ni uniformes, mais le plus généralement constitués par l'agglomération de structures plus fines. Dans de petites régions localisées, les grains semblent systématiquement plus petits, ne dépassant pas $0 " 6$. Le contraste des grains, mesuré photométriquement à l'Observatoire de Sacramento Peak, a été trouvé de l'ordre de I5 à $20 \%$.

\section{SUGGESTIONS DES MEMBRES DE LA COMMISSION}

I. Examiner à nouveau la question du perfectionnement des méthodes qui permettent de chiffrer rapidement l'activité solaire, cette question n'ayant pas reçu de solution complète au Congrès précédent. Comme, d'autre part, il apparaît clairement, à l'heure actuelle, que les observations photosphériques ne peuvent plus être séparées de celles de la chromosphère, du champ magnétique solaire et des phénomènes radioélectriques, cette question devrait être examinée conjointement avec les commissions II, I2 et 40 (Uccle).

2. Maintenir le programme traditionnel d'observation des taches et des facules en en affinant les critères et développer le programme nouveau proposé par Zurich en I948, en portant une attention particulière aux observations photographiques de l'évolution rapide des détails au sein des groupes de taches et à l'étude de petites régions polarisées, au moyen d'instruments à long foyer et dans de bonnes conditions de stabilité des images (Uccle).

3. Favoriser dans l'avenir, quand l'A.G.I. sera terminée, les transmissions rapides par voie télégraphique des renseignements sur l'activité solaire. Un service central des ursigrammes devrait être organisé sous les auspices des trois Unions: U.R.S.I., U.G.G.I. et U.A.I. (Uccle).

4. Reprendre l'idée exprimée au précédent Congrès d'organiser une étude en commun de la granulation photosphérique avec des instruments standardisés et dans des régions spectrales variées (Macris).

Ces quatre questions auront la priorité dans l'Ordre du jour de la séance de la Commission. Celle-ci aura en outre à examiner la proposition suivante, formulée par l'Observatoire fédéral de Zurich:

'La Commission recommande que la subvention annuelle de Iooo francs or accordée précédemment à l'Observatoire de Zurich pour la publication des Cartes héliographiques de la Photosphère, soit renouvelée pour la période qui s'étendra jusqu'à la prochaine Assemblée générale.'

\section{D' A Z A M B U J A \\ Président de la Commission}

\section{REFERENCES}

[I] Newton, H. W. et Milsom, A. S. Mon. Not. R. Ast. Soc. I15, 398, I955.

[2] Mursalinova, G. G. Publ. Tashkent Astr. Obs. 5, 145, 1956.

[3] Kopecký, M. Bull. Astr. Insts. Czechosl. 4, 125, 1953; 6, 17, 1955; Publ. Astr. Inst. Acad. Sci. Czechosl. no. 28, 1956.

[4] M. Kopecký, Bull. Astr. Insts. Czechosl. 4, 67, 1953; 7, 65, 1956.

[5] Kopecký, M. et Mayer, P. Bull. Astr. Insts. Czechosl. 5, r32, 1954.

[6] Hotinli, M. Publ. Istanbul Univ. Obs. no. 56, 1955.

[7] d'Azambuja, Mme M. C.R. Acad. Sci., Paris, 241, I71 2, 1955.

[8] Jensen, E., Nordö, J. et Ringnes, T. S. Astrophys. Norveg. 5, I67, 1955.

[9] Tandberg-Hanssen, E. Astrophys. Norveg. 5, 207, 1956.

[10] Gleissberg, W. Publ. Istanbul Univ. Obs. no. 53, 1954.

[II] Waldmeier, M. Z. Ap. 43, 29, I957; Astr. Mitt., Zürich, no. 207. 


\section{PHENOMENES PHOTOSPHERIQUES}

[1 2] Kopecký, M., Kvičala, J. et Ptáček, J. Bull. Astr. Insts. Czechosl. 8, 106, 1957.

[13] Waldmeier, M. Z. Ap. 43, 149, 1957; Astr. Mitt., Zürich, no. 209.

[14] Milosević, K. C.R. Acad. Sci., Paris, 240, 731, 1955; 24I, 59o, r955.

[15] Becker, U. Z. Ap. 37. 47, 1955.

[16] Gleissberg, W. Istanbul Univ. Obs. Yazil, no. 5I, $219,1954$.

[17] Gleissberg, W. A. N. 283, 23, 1956.

[18] Nicholson, S. B. Astr. J. 60, I74, I955.

[19] Slonim, J. M. et Kuleshova, K. F. Publ. Tashkent Obs. 6, Ior, 1957.

[20] Fortini, T. R.C. Accad. Lincei, 19, 131, 1955; 19, $272,1955$.

[21] Djurković, P. M. Solnetchnie Danie A.N. S.S.S.R. 1, 6, I954.

[22] Christjakov, V. F. Djull. vsesoj ast. geod. Obshch. S.S.S.R. no. 13, 29, I953.

[23] Arbey, L. Bull. Astr., Paris, 20, 347, 1956.

[24] Vitinsky, U. I. et Rubashev, B. M. Solar Data, no. 2, II4, 1957.

[25] Xanthakis, J. Astr. Zh. S.S.S.R. T. 34 B, 1957.

[26] Waldmeier, M. Z. Ap. 43, 149, 1957; Astr. Mitt., Zürich, no. 209, 1957.

[27] Schove, D. J. J. Geophys. Res. 60, I27, I955; J. Brit. Astr. Ass. 66, 59, 1956.

[28] Gleissberg, W. Istanbul Univ. Fen. Fac. Mecm. sér. C, no. 20, 263, I955.

[29] Dizer, M. Ann. Astrophys. 19, 207, 1957.

[30] Thuring, B. A. N. 282, 33, 1955.

[3I] Ogawara M. et Tomatsu, K. Pap. Met. Geophys., Tokyo.

[32] Kopecký, M. Bull. Astr. Insts. Czechosl. 7, 5, 1956.

[33] Gleissberg, W. Die Sterne, 32, 72, 1956.

[34] Gleissberg, W. Istanbul Univ. Obs. Yazil, no. 59, 1957.

[35] Alfvén, H. Tellus, 8, 274, 1956.

[36] Chopra, K. P. Publ. Astr. Soc. Japan, 8, 55, 1956.

[37] Menzel, D. H., Layzer, D. et Krook, M. Astr. J. 60, 171, 1955.

[38] Parker, E. N. $A p . J$. 121, 491, I955.

[39] Stepanov, V. E. et Kljakotko, M. A. Publ. Crim. Obs. 16, 80, I956.

[40] Miller, W. A. Nature, 155, 557, 1955.

[4r] Stepanov, V. E. Publ. Sternberg astr. Inst., roo.

[42] Thiessen, G. Z. Ap. 35, 237, 1954.

[43] Krat, V. A. et Goldberg-Rogozinskaya, N. M. Pulkovo Bull. no. 155, 1956.

[44] Macris, C. et Elias, D. Ann. Astrophys. 18, 143, 1955.

[45] Miller, W. A. Astr. J. 6r, $187,1956$.

[46] Stuart, F. E. et Rush, J. H. Ap. J. 120, 245, 1954.

[47] Skumanich, A. Ap. J. 121, 404, 1955.

[48] Wlérick, G. Smithson. Contr. Astrophys. 2, 25, 1957.

[49] Uberoi, M. S. Ap. J. 121, 400, 1955; 122, 466, 1955.

[50] Rösch, J. C.R. Acad. Sci., Paris, 240, 1630, 1955; 243, 478, 1956; 243, 2020, 1956; L'A stronomie, 7 rme année, r 29, 1957.

[5I] Blackwell, D. E., Dewhirst, D. W. et Dollfus, A. Nature, 180, 2 I I, 1957.

\section{Compte rendu de la Séance. I4 août 1958}

Président: L. d'Azambuja.

SECRÉTAIRE: M. Kopecký.

En ouvrant la séance, le Président demande aux membres présents s'ils ont quelque remarque ou objection à présenter sur le Draft Report qu'il a préparé.

M. Cimino signale que les observations du Monte-Mario, publiées auparavant dans les Memorie della Società astronomica italiana et indiquées dans le Rapport comme insérées dans ce périodique, font l'objet d'une publication indépendante depuis le rer janvier dernier. Il sera tenu compte de cette remarque dans le Draft Report, qui est alors adopté sans autre modification. 


\section{COMMISSION 10}

Chiffrement de l'activité solaire. Cette question a été inscrite à l'ordre du jour à la demande de l'Observatoire d'Uccle. Aucun représentant de cet établissement n'étant présent à la séance, il ne paraît pas opportun d'aborder ce sujet. M. d'Azambuja rappelle simplement que celui-ci figurait déjà au programme de la Commission lors de sa précédente réunion à Dublin (Trans. I.A.U. 9, I33, I955) et que, sans qu'une décision ferme soit prise, diverses solutions avaient été envisagées, parmi lesquelles celle suggérée par Mme d'Azambuja, consistant à totaliser les aires des taches visibles à un même moment sur le disque, en les déduisant simplement de la longueur du plus grand diamètre de chaque tache, considérée comme approximativement circulaire. Le nombre ainsi obtenu, aisément et rapidement, serait en plus étroite correspondance avec la surface tachée que le nombre de Wolf.

Evolution rapide des détails des taches. Le Président regrette l'absence de M. Waldmeier, Directeur de l'Observatoire de Zurich, qui a organisé une coopération internationale pour cette étude et qui aurait pu donner des détails intéressants sur les résultats déjà acquis.

M. d'Azambuja attire l'attention sur des travaux récents effectués par M. Rösch à l'Observatoire du Pic-du-Midi avec une lunette de $23 \mathrm{~cm}$ d'ouverture munie d'une caméra cinématographique permettant de prendre jusqu'à 25 images par seconde, et qui montrent combien l'agitation de l'air peut changer d'une manière considérable l'aspect des détails des taches en l'espace d'une fraction de seconde. En raison de ces déformations, les détails observés n'ont été retenus comme réellement solaires que lorsqu'ils se retrouvaient identiques à eux-mêmes sur deux poses séparées par quelques secondes.

M. Rösch estime qu'il serait important d'utiliser une technique de ce genre pour toute étude de l'évolution des taches. Ainsi, au lieu d'effectuer une photographie unique toutes les trois heures, comme il a été convenu entre les stations participant à la coopération internationale, il serait plus efficace de remplacer cette photographie par une série de poses s'étendant sur une à deux minutes et comportant des intervalles de temps de l'ordre de ceux du Pic-du-Midi.

M. Roberts fait observer qu'il est difficile, pendant l'A.G.I., de modifier le programme établi par Zurich.

M. Djurković craint en outre que l'équipement des différentes stations soit difficile à standardiser complètement. Il indique que la station de Belgrade, pour éviter précisément les effets de la turbulence, a réalisé un dispositif qui ne permet à la lumière solaire d'entrer dans l'instrument qu'au moment même de la pose.

M. d'Azambuja s'offre à mettre M. Waldmeier au courant de cette discussion et à lui demander son avis sur la suggestion de M. Rösch. Aucune objection n'est faite par la Commission à cette proposition.

Ursigrammes. L'Observatoire d'Uccle, insistant sur l'intérêt des transmissions rapides, par voie télégraphique, des renseignements sur l'activité solaire, propose qu'après l'Année géophysique internationale, un service central des ursigrammes soit organisé sous les auspices des trois Unions: U.R.S.I., U.G.G.I. et U.A.I.

Les ursigrammes donnant essentiellement des informations sur les phénomènes chromosphériques et coronaux, cette proposition est transmise à la Commission des couches extérieures du Soleil.

Granulation photosphérique. M. Rösch présente des photographies, obtenues par le procédé indiqué plus haut pour les taches, et montrant des granules intra-maculaires dont la durée de vie paraît être supérieure à ro min (intervalle de temps maximum des clichés disponibles), ainsi que des granules photosphériques, dont la durée de vie est peut-être supérieure à la valeur admise jusqu'ici.

M. Krat suggère que la longue durée de vie signalée par M. Rösch est peut-être due à des variations lentes de la réfraction à l'intérieur de l'instrument. Il estime que, pour ce genre de recherches, les réflecteurs sont supérieurs aux réfracteurs.

M. Dollfus rend compte des observations que, en collaboration avec MM. Blackwell et Dewhirst, de l'Observatoire de Cambridge (Angleterre), il a effectuées en ballon libre (voir p. I59) à l'aide d'une lunette photographique de $30 \mathrm{~cm}$ d'ouverture et à $6000 \mathrm{~m}$ d'altitude. 


\section{PHENOMENES PHOTOSPHERIQUES}

Il signale que M. Schwarzschild a fait peu après, avec l'aide de divers organismes des Etats-Unis, des observations analogues avec un télescope de $30 \mathrm{~cm}$ également, pointé automatiquement sur le Soleil et porté par un ballon sans équipage. L'étude photométrique des clichés obtenus, ainsi que le tracé des courbes de corrélation entre des paires de points de distances croissantes apportent de nouveaux et nombreux renseignements qui ont été exposés au symposium sur les observations astronomiques en ballon, fusées et satellites artificiels (voir pp. 705-6).

M. Krat remarque que les photographies obtenues dans les deux expéditions en ballon coïncident entre elles, mais diffèrent de celles obtenues au sol par l'Observatoire du Pic-duMidi.

Subvention. Le Président propose de soumettre à l'Assemblée générale la recommandation suivante, présentée par M. Waldmeier:

$\mathrm{La}$ Commission recommande que la subvention annuelle de Iooo fr.or, accordée précédemment à l'Observatoire de Zurich pour la publication des Cartes héliographiques de la Photosphère, soit renouvelée pour la période qui s'étendra jusqu'à la prochaine Assemblée générale.

La recommandation est adoptée par la Commission.

Avant de lever la séance, M. d'Azambuja indique qu'il a décidé de cesser toute activité scientifique officielle; il remercie les membres de la Commission pour la coopération fructueuse qu'ils n'ont cessé de lui apporter pendant les six années de sa présidence. 